\title{
Development and Testing of Digital Mammography Displays and Workstations
}

National Cancer Institute

\section{Source}

National Cancer Institute. Development and Testing of Digital Mammography Displays

and Workstations. NCI Thesaurus. Code C15948.

This initiative is intended to advance the state-of-the-art in digital mammography displays and workstation design to facilitate clinical acceptance and implementation of digital mammography for improved breast cancer diagnosis. This PA will solicit research and development in three critical areas in digital mammography: 1) Softcopy Display Hardware; 2) Workstation Software and Design; and 3) Image Perception. 